\title{
Combined DCT-Haar Transforms for Image Compression
}

\author{
Issam Dagher, Mireille Saliba, Rachelle Farah \\ Department Of Computer Engineering, University Of Balamand \\ POBOX 100, Elkoura, Lebanon \\ dagheri@balamand.edu.lb
}

\begin{abstract}
Discrete Cosine Transform (DCT) and Haar wavelet transform are very important transforms in image compression. While DCT works extremely well for highly correlated data, the Haar transform gives superior results for images exhibiting rapid gradient variations. The objective of this paper is to combine the advantages of these 2 transforms into one transform in order to get better peaksignal-to-noise-ratio (PSNR) and keeping good compression ratio. Following the JPEG, our first approach was to apply separately on each $8 \times 8$ block the Haar and the DCT transforms. A signaling bit along the transform which gave better PSNR are quantized and transmitted. This approach gave better PSNR than each one separately. Our second approach was to construct a new hybrid transform which is a combination of these 2 transforms. We mixed both DCT and Haar transforms into one transform. We derived the new transform coding formulas. We applied our hybrid transform on each block obtained. Results show that our approach outperforms the existing DCT and Haar methods, keeping good quality of the image even for high compression ratios, giving a higher PSNR than DCT for the same compression ratio, and permitting better edge recovery than the Haar transform.
\end{abstract}

Keywords: Image Compression, DCT, Haar, Wavelet, Hybrid.

\section{Introduction}

Image compression is an efficient technique of reducing the size of data representing an image that is highly correlated. The purpose of image compression is to save memory and transmission time [1]. There are different methods in which image can be compressed. One of the most common compressed graphic image formats is the JPEG format which is based on the Discrete Cosine Transform (DCT) [2]. Another most commonly transform used in image compression is the Wavelet transform. One of the simplest wavelet is the Haar wavelet. It is based on doing average and difference operations on the image intensity values [6]. There are two kinds of compression [10]. Lossless compression involves the preservation of the image as if no detail is lost. Lossy compression, allows less than perfect reproduction of the original image. The advantage of lossy compression (our work) is that it can produce a much smaller compressed file than lossless compression. Note that JPEG applies DCT with a zigzag quantization that makes the compression visually lossless. JPEG 2000 [3] uses the Wavelet Transform in their compression scheme. DCT is well suited for highly correlated data. It does a kind of de-correlation [7] for those data and has the property of energy compactness. Energy compactness means that DCT tries to concentrate energy or information in some elements of the block instead of spreading it in the whole block. This helps in increasing the compression since we are only interested in the elements that contain the maximum energy needed. Note that DCT concentrates this energy in the upper left corner of the block where the low frequencies reside. On the other hand, the Haar transform [8] works well for high frequencies and achieves a high compression ratio while maintaining the quality of the image. It is very well performant for images that have rapid gradient variations.Combining DCT and DWT was done in [12] [13] by applying the discrete cosine transform on the discrete wavelet transform coefficients. In [11] Hybrid matrices are generated using combination of various matrices like DCT, Hadamard and Kekre's Transform.

\subsection{JPEG}

The JPEG (Joint Photographic Experts Group) algorithm was founded to compress images with minimal data loss and high compression ratios. The JPEG consists of 4 different phases. First, the image is divided into 8x8 blocks of pixels. Next, the DCT can be applied to each block of image to convert it to the frequency domain. Then each one is compressed by performing a quantization process to remove the unnecessary information. Then, the transform coefficients which are 
quantized are transmitted to the receiver. Therefore, the DCT plays an important role of compression in the JPEG standard that offers a lossy compression of images.

\subsection{DCT}

The discrete cosine transform (DCT) is a concept for converting a signal into elementary frequency components and is based on dividing images into parts of different frequencies. The DCT de-correlates the image data. The de-correlation is the removal of redundancy between adjacent pixels [5]. It is one of the useful properties of Discrete Cosine Transform. After applying the DCT, the autocorrelation becomes very small. The data becomes uncorrelated. We can remove the unnecessary coefficients (small values) and transform the coefficients orderly to encode them independently and uniquely without repeating the same information represented in correlated pixels twice or more. Equation 1 shows an example of the equation of the Discrete Cosine Transform (DCT)

$$
g^{\prime}(x, u)=\lambda(u) \cos \frac{\pi(2 x+1) u}{2 N}
$$

where $\lambda(u)= \begin{cases}\sqrt{\frac{2}{N}} & u=0 \\ \sqrt{\frac{1}{N}} & \text { otherwise }\end{cases}$

\subsection{Wavelet Transform}

Another very popular technique in image compression is the Discrete Wavelet Transform. The purpose of Wavelet transform is to change the data from time-space domain to time-frequency domain for a better compression. In addition, The Discrete Wavelet Transform can give high compression rate [4]. First, the image is passed into two filters: a high $(\mathrm{H})$ and a low (L) pass filter. This process can be repeated in order to get the four sub-bands LL, HL, LH and HH. This is called the 2D DWT [9].The Wavelet Transform produces a large number of zero or near zero coefficients. The compression is done by thresholding these values.

\subsubsection{Haar Transform}

The Haar transform is a part of the wavelet family. It has its own basis and is the simplest wavelet basis. Equation 2 shows the Haar Transform basis formulas.

$$
\Psi(\mathrm{x})=\left\{\begin{array}{c}
1,0.0 \leq x<0: 5 \\
-1,0.5 \leq x<1: 0 \\
0, \quad \text { otherwise }
\end{array} \quad \emptyset p q(x)=2^{p / 2} \Psi\left(2^{p} x-q+1\right)\right.
$$

\subsection{PSNR}

It is regularly necessary to proceed with some measures to quantify the quality of reconstruction of lossy compression. The Peak Signal-to-Noise Ratio (PSNR) is one of these measures [14]. It is given by the following equation:

$$
P S N R=20 \log _{10}\left(\frac{255}{\sqrt{\frac{1}{n * m} \sum_{i=0}^{n-1} \sum_{j=0}^{m-1}\left[f(i, j)-f^{\prime}(i, j)\right]^{2}}}\right)
$$

where $n x m$ is the size of the image, $f(i, j)$ is the pixel of row $i$ and column $j$ of the original image and $f^{\prime}(i, j)$ is the pixel of row $\mathrm{i}$ and column $\mathrm{j}$ of the reconstructed image 


\section{A Hybrid DCT-Haar Block Compression \\ 2.1. Method Concept}

DCT and Haar are well known and used compression transformations especially in the well-known JPEG compression. Our method is based on combining both of them. It will achieve a better peak signal to noise ratio PSNR keeping good compression ratio.

The method consists of the following steps:

- Dividing the image into 8x8 blocks

- Computing the DCT and Haar transforms for each block

- Applying a certain quantization

- Computing the inverse transform of each result

- Getting the PSNR of both results

- Sending the block with the highest PSNR

- Sending a signaling bit indicating the type of the transform (DCT or Haar).

Once the receiver gets the block, it does the corresponding inverse transform based on the signaling bit received.

\subsection{Energy Compactness and Quantization}

Energy compactness and quantization (or simply in this paper we call it quantization) is used to take the useful information from the $8 \times 8$ block. The remaining information is put to zero in order not to be sent to the receiver. Quantization is applied differently on DCT and Haar blocks.

\subsubsection{DCT Quantization}

The DCT transform concentrates the low frequencies that contain useful information in the upper left corner of the block. DCT quantization consists on keeping the low frequencies and zeroing the other entries. Therefore, in $8 \times 8$ blocks, mxm subblock in the upper left corner of the block is kept while the other entries are put to zero.

\subsubsection{Haar Quantization}

After applying the Haar transform, the values that are less than a threshold $\alpha$ are zeroed. The remaining values are kept. A small compression ratio leads to a small compression. However, the PSNR will be better

\subsection{Compression Ratio}

After applying the quantization, the compression ratio must be calculated to see the efficiency of the algorithm. To do a fair comparison between the different algorithms, we have calculated it in the following manner for an 8-bit monochrome image.

For the DCT blocks, the number of zero elements must be counted and multiplied by 8 . Then the number of the DCT blocks must be subtracted from the result. This is done because a signaling bit is sent to tell the nature of the transform applied to the block.

The same is applied for the Haar transform. In addition, for non-zero elements, their place in the block should be sent so that the receiver knows where to place the entry. Therefore, 6 additional bits are needed to specify the row and column of each non-zero entry. In fact, we have 8 rows, so 3 bits are needed to encode the row number. The same applies for the column. Therefore, the product of 6 and the number of non-zero elements should be subtracted from the previous result. A diagram of the process is shown in Figure 1. 


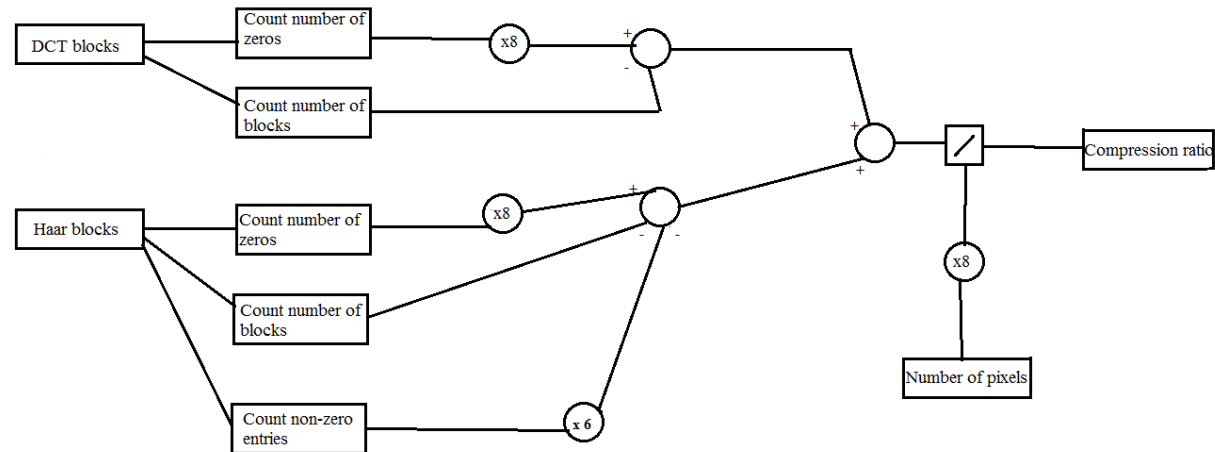

Fig. 1: Compression Ratio Calculation.

The values obtained from DCT and Haar blocks should be added. Let R be the result. The compression ratio will be as shown in Equation 4.

$$
\text { Compression ratio }=\frac{R}{8 * \text { number of pixels in the image }}
$$

\subsection{Simulation and Results}

To validate our proposed methods, we used a set of 4 greyscale images: Barbara, Boat, Peppers and Lena that are represented respectively in Figure 2 . The size of each image is $512 \times 512$.

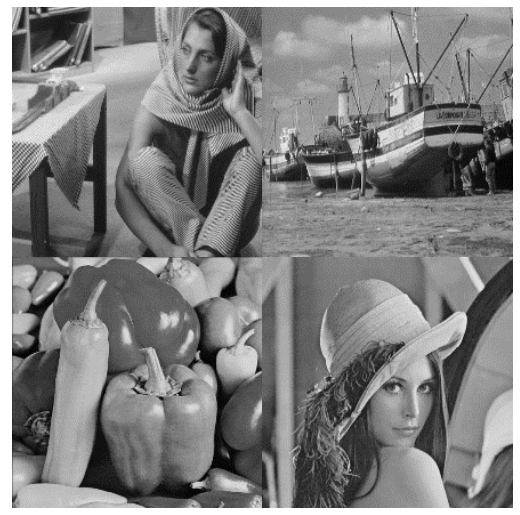

Fig. 2: Images used.

The efficiency of our method is tested in this section. It must be compared with the DCT and Haar transforms applied on $8 \times 8$ blocks using the same quantization method.

In order to compare our method with the DCT transform, different values for the parameter $m$ are used and the PSNR value for each $m$ is calculated. For the Haar blocks chosen in our method, $\alpha$ is set to 15 . Plots of $m$ versus PSNR values for different images are shown in Figure 3. 

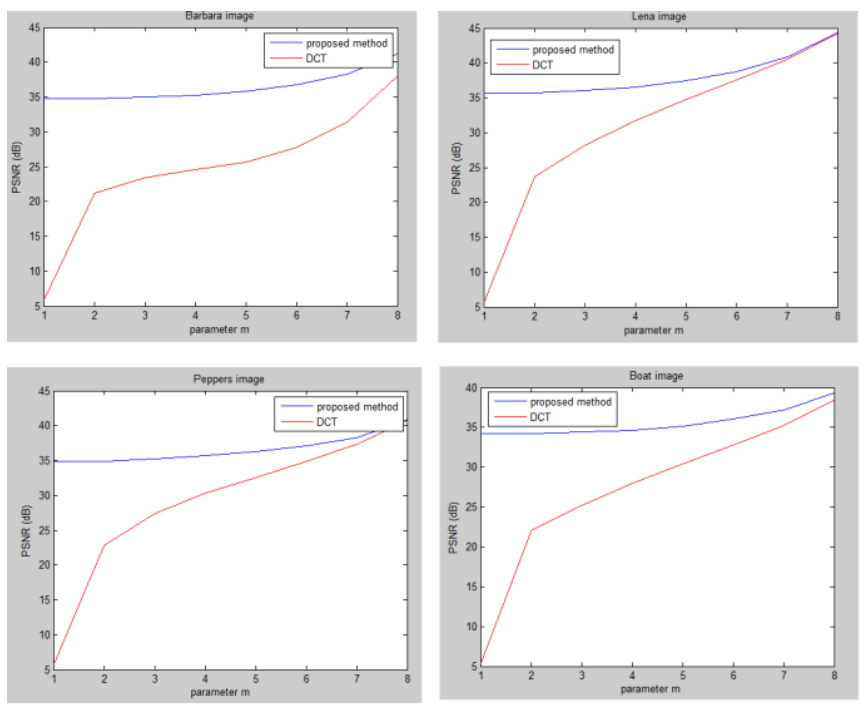

Fig. 3: DCT Parameter m vs PSNR for Different Images Using DCT and our Method.

It is clear that our method gives a higher PSNR than DCT for the same value of $m$ for those images especially when there is more compression. In fact a small value of $\mathrm{m}$ means that a bigger portion of the block is set to zero i.e. more compression is involved. More compression means a smaller PSNR value.

In order to compare our method with the Haar transform, different values for the parameter $\alpha$ are used and the PSNR value for each $\alpha$ is calculated. For the DCT blocks chosen in our method, $m$ is set to 4 . Plots of $\alpha$ versus PSNR values for different images are shown in Figure 4.
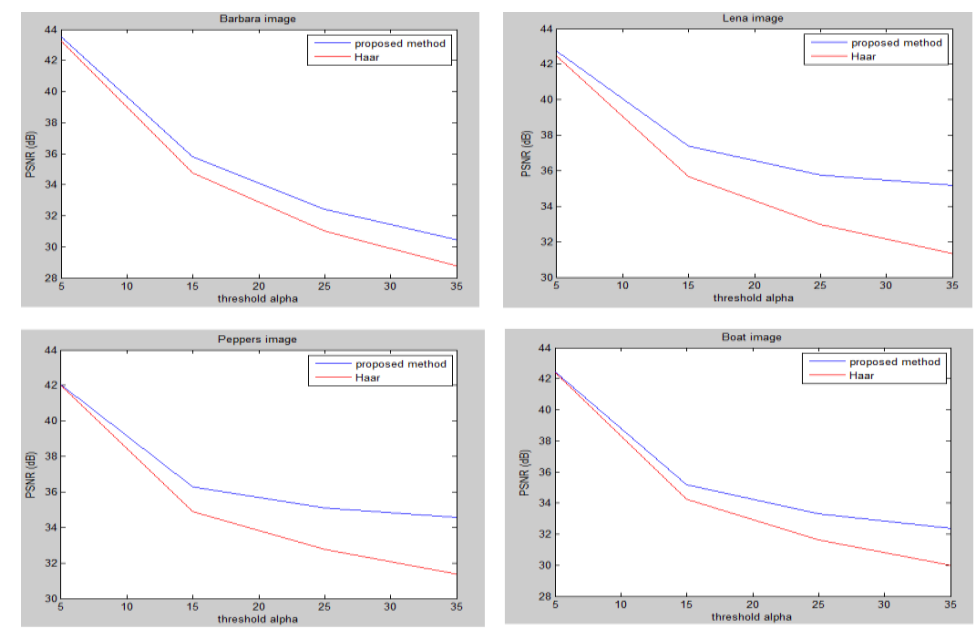

Fig. 4: Haar Threshold $\alpha$ vs PSNR for Different Images Using Haar and our Method.

It is clear that our method gives a higher PSNR than Haar for the same value of $\alpha$ for those images especially when there is more compression. In fact a large value of $\alpha$ means that a bigger portion of the block is set to zero i.e. more compression is involved. More compression means a smaller PSNR value.

The results show that our method outperforms the DCT and Haar transforms for those four images. In addition, for the Barbara image where there are a lot of high frequencies due to the big number of edges, it is clear that our method has a better recovery than DCT and Haar.

To investigate more, DCT and our method are applied for the 4 images for the same compression ratio as shown in Figure 5. 

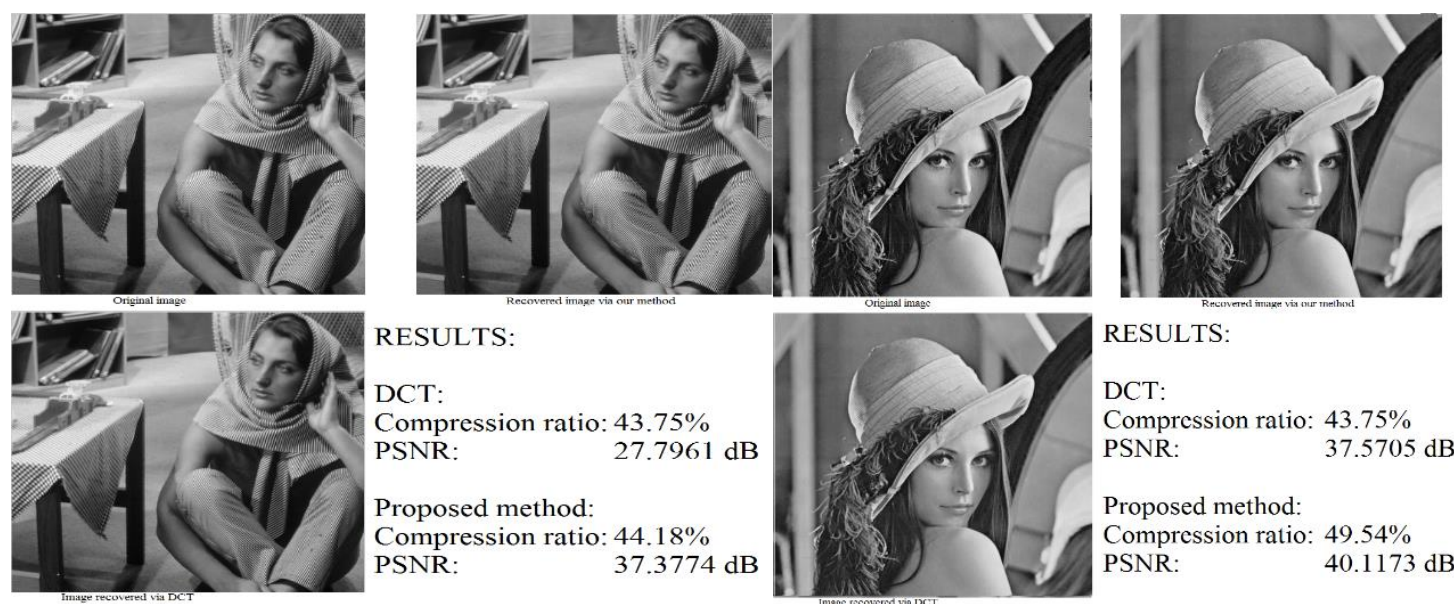

RESULTS:

DCT:

Compression ratio: $43.75 \%$

PSNR:

$37.5705 \mathrm{~dB}$

Proposed method:

Compression ratio: $49.54 \%$

PSNR: $\quad 40.1173 \mathrm{~dB}$

(a)

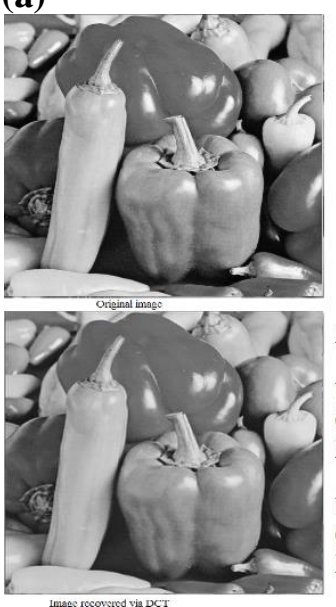

(c)

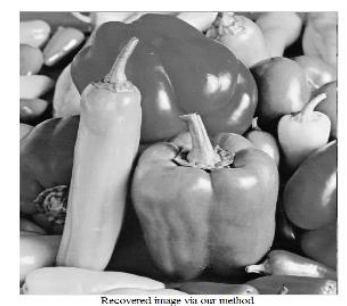

RESULTS:

DCT:

Compression ratio: $60.94 \%$

PSNR:

Proposed method:

Compression ratio: $60.32 \%$

PSNR: $\quad 36.2902 \mathrm{~dB}$ (b)
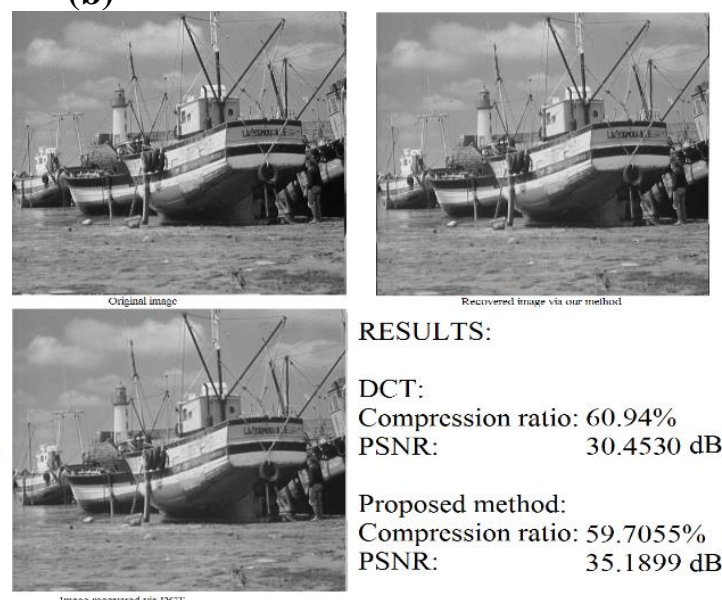

RESULTS:

DCT

Compression ratio: $60.94 \%$

PSNR: $\quad 30.4530 \mathrm{~dB}$

Proposed method:

Compression ratio: $59.7055 \%$

PSNR: $\quad 35.1899 \mathrm{~dB}$

(d)

Fig. 5: Results for the Barbara (a), Lena (b), Pepper(c), and Boat (d) images.

Figure 5 shows that our method gives a PSNR better than that of DCT for the same compression ratio.

Also the error of recovery via the Haar transform in all images is bigger than the error produced from our method. The error reflects the ability to recover the images' edges. Therefore, our method gives a better PSNR than DCT and better edge recovery than Haar. In order to see the performance of our method, the thresholds $\alpha$ of Haar and m of DCT are varied. This is accompanied by presenting the percentage of the sent DCT blocks for the Pepper image. Results are shown in Table 1.

Table 1: Variation of $\alpha$ and $m$ for Pepper Image.

\begin{tabular}{|l|l|l|l|l|}
\hline Threshold $\alpha$ for Haar blocks & $\mathbf{5}$ & $\mathbf{1 5}$ & $\mathbf{2 5}$ & $\mathbf{3 5}$ \\
\hline $\mathbf{m = 2}$ (for DCT blocks) & 1.147461 & 53.78418 & 68.9209 & 75.56152 \\
\hline Percentage of DCT blocks & 41.18938 & 78.53937 & 82.9937 & 84.62334 \\
\hline Compression ratio (\%) & 41.96088 & 35.22682 & 33.40736 & 32.26997 \\
\hline PSNR (dB) & \multicolumn{3}{|l|}{} \\
\hline m=3 (for DCT blocks) & 3.271484 & 68.79883 & 80.98145 & 86.25488 \\
\hline Percentage of DCT blocks & 40.81726 & 71.31433 & 74.52049 & 75.40245 \\
\hline Compression ratio (\%) & 41.97367 & 35.67078 & 34.15795 & 33.32225 \\
\hline PSNR (dB) & & & \\
\hline m=4 (for DCT blocks)
\end{tabular}




\begin{tabular}{|l|l|l|l|l|}
\hline Percentage of DCT blocks & 7.446289 & 79.1748 & 88.5498 & 92.38281 \\
\hline Compression ratio (\%) & 39.48746 & 60.32076 & 62.00457 & 62.18891 \\
\hline PSNR (dB) & 42.00663 & 36.29016 & 35.11305 & 34.56648 \\
\hline m=6 (for DCT blocks) & 25.24414 & 92.84668 & 96.99707 & 97.90039 \\
\hline Percentage of DCT blocks & 28.40385 & 25.55332 & 24.9011 & 24.56732 \\
\hline Compression ratio (\%) & 42.3059 & 38.3067 & 37.9219 & 37.809 \\
\hline PSNR (dB)
\end{tabular}

Table 1 shows that for any value of $\mathrm{m}$, increasing $\alpha$ leads to more compression. However, for the same $\alpha$ increasing $\mathrm{m}$ leads to less compression. Compression ratio and PSNR are inversely proportional. In fact, increasing the compression leads to a sacrifice in the image quality. The percentage of the DCT blocks taken is affected by the values of $\alpha$ and $\mathrm{m}$. Increasing $\alpha$ for a fixed $m$ leads to take more DCT blocks because big values of $\alpha$ lead to a high compression and low PSNR for Haar blocks. This makes the sender choose more DCT blocks. For a fixed value of $\alpha$, decreasing $m$ leads to choosing more Haar blocks. This can be explained in the same manner as before.

Overall, examining the values of the table can help in choosing the best $\alpha$ and $\mathrm{m}$ to be applied in the algorithm. For $\alpha=15$ and $m=4$, the compression ratio is acceptable and the PSNR value indicates a good quality of the image. Therefore, those values (for $8 \times 8$ blocks) are chosen to be applied in the compression algorithm of our method.

\section{Conclusion}

This paper showed that combining DCT and Haar transforms gives better results than using each one alone. It tried to take benefit of the advantages of both transforms while minimizing the effects of the weaknesses of each one of them. Results showed that for the same parameter m of the DCT transform, our method always gives a higher PSNR than the DCT transform applied alone. For the same threshold, our method always gives better edge recovery. Therefore, our method increases the PSNR obtained by the DCT transform and enhances the edge recovery of the Haar transform.

\section{References}

[1] A. Swaminathan, G. Agarwal, "A comparative study of image compression methods," Digital Image Processing, course project, 2001.

[2] B. Nilesh, S. Sachin, N. Pradip, D. Rane, "Image compression using discrete wavelet transform," International Journal of Computer Technology and Electronics Engineering (IJCTEE)., vol. 3, pp. 85-89, 2013.

[3] A. Kaur, J. Kaur, "Comparison of DCT and DWT of image compression techniques," International Journal of Engineering Research and Development, vol. 1, no. 4, pp. 49-52, 2012.

[4] E. Ramandeep, "Image compression using discrete cosine transform and discrete wavelet transform," International Journal of Computing and Business Research., 2012.

[5] J. J. Ding, Y. W. Huang, P. Y. Lin, S. C. Pei, H. H. Chen, Y. H. Wang, "Two-dimensional orthogonal DCT expansion in trapezoid and triangular blocks and modified JPEG image compression," Image Processing, IEEE Transactions on, vol. 22, no. 9, pp. 3664-3675, 2013.

[6] A. Kapoor, R. Dhir, "Image Compression Using Fast 2-D DCT Technique," International Journal on Computer Science and Engineering, vol. 3, no. 6, pp. 2415-2419, 2011.

[7] P. Kapoor, S. Kumara, G. Kaur, "A review on image compression," International Journal of Advanced Research in Computer Science and Software Engineering, vol. 4, no. 9, pp. 128-132, 2014.

[8] P. Dixit, M. Dixit, "Study of JPEG image compression technique using discrete cosine transformation," International Journal of Interdisciplnary Research and Innovations, vol. 1, no. 1, pp. 32-35, 2013.

[9] E. Agrawal, K. Manu, R. Varshney, A. Yadav, "DCT and systolic array in image compression," International Journal of Research in Engineering and Technology, vol. 3, no. 6, pp. 77-80, 2015. [Online]. Available: http://oaji.net/articles/2015/489-1437044954.pdf

[10] O. Hunt, R. Mukundan, "A comparison of discrete orthogonal basis functions for image compression," University of Canterbury, Dept. Computer Science \& Software Engineering, 2004. [Online]. Available: http://ir.canterbury.ac.nz/handle/10092/447 
[11] N. R. Thota, S. K. Devireddy, "Image compression using discrete cosine transform," Georgian Electronic Scientific Journal: Computer Science and Telecommunications, vol. 3, no. 17, pp. 35-43, 2008.

[12] S. A. Khayam, "The discrete cosine transform (dct): theory and application," Michigan State University, vol. 114, 2003.

[13] P. Xiao, "Image Compression by Wavelet Transform," M.S. Thesis, East Tennessee State University, Johnson, TN, 2001 (unpublished).

[14] E. Shusterman, M. Feder, "Image compression via improved quadtree decomposition algorithms," Image Processing, IEEE Transactions On, vol. 3, no. 2, pp. 207-215, 1994.

[15] P. Strobach, "Image coding based on quadtree-structured recursive least-squares approximation," International Conference on Acoustics, Speech, and Signal Processing, ICASSP-89, pp. 1961-1964, 1989.

[16] W. G. Aref, H. Samet, "Decomposing a window into maximal quadtree blocks," Acta Informatica, vol. 30, no. 5, pp. 425-439, 1993.

[17] A. Neri, M. Carli, V. Palma, L. Constantini, "Image search based on quadtree Zernike decomposition," Journal of Electronic Imaging, vol. 19, no. 4, p. 043023, 2010. 